\title{
THE EXTRAGALACTIC RADIO SOURCE BACKGROUND
}

\author{
J. V. Wall \\ Royal Greenwich Observatory \\ Isaac Newton Group of Telescopes \\ Apartado 321, 38780 Santa Cruz de La Palma \\ Tenerife, Spain
}

\begin{abstract}
The background of discrete extragalactic radio sources is described from the point of view of its simplest properties: surface density and surface distribution. New considerations, particularly with regard to the latter, open the possibility of detecting Universal structure on many angular scales. At the same time, these considerations imply the need for greater sophistication in the interpretation of source counts and of the microwave background (MWBG) fluctuations.
\end{abstract}

\section{BACKGROUND TO THE BACKGROUND}

My task is to discuss the background of extragalactic radio sources, leaving the microwave radio background itself to others. However, the organizers requested that $I$ include something of the long-wavelength background, and to do so I must tell you the following short story.

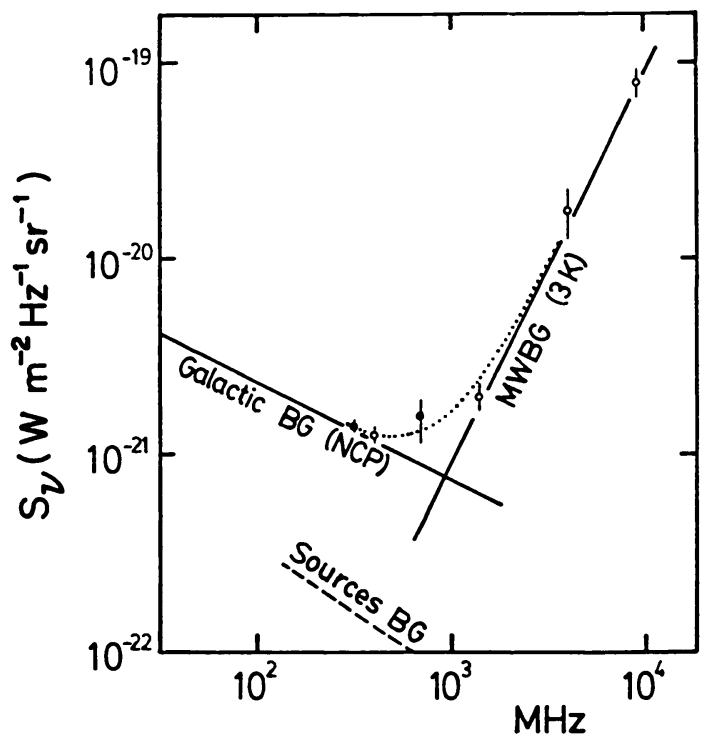

Figure 1. Sky brightness vs. frequency; the dotted curve represents the total sky brightness, galactic background plus a thermal $3 \mathrm{~K}$, while the dashed curve is the integrated background due to discrete sources. The circles are experimental results.
Once upon a time (1964) two graduate students at the University of Toronto were charged by their supervisor, J. L. Yen, with measuring the absolute background on large scales at two frequencies - 320 and $707 \mathrm{MHz}$. This they did (and very well, I might add). But from a comparison of the two independent sky maps, the poor graduate students obtained a spectral index of about -0.3 . Everybody knew - from scaled-antenna (non-absolute) measurements - that the spectral index of the galactic radio background was -0.6 or -0.8 . Were the zero levels wrong? Was something added everywhere?

Yes it was. You will have guessed the answer, which was supplied by Penzias and Wilson (1965). When we (Wall, Chu and Yen 1970) subtracted a $3^{\circ}$ background from our results, all became clear, as Figure 1 shows. 
Figure 1, in fact, indicates how the large-scale radio background is composed. Synchrotron radiation from the Galaxy (polluted somewhat by thermal radiation from HII regions in the plane) dominates up to about $1 \mathrm{GHz}$; then the thermal $3^{\circ} \mathrm{K}$ of the MWBG takes over. In terms of large-scale sky emission, the background of extragalactic radio sources hardly figures at all, although large-scale maps such as those of Wall, Chu and Yen (1970) are indeed distorted by the very brightest extragalactic sources.

Nevertheless, that background of discrete extragalactic sources found in the course of sky surveys has contributed fundamentally to our understanding of astrophysics and cosmology. I propose to discuss two aspects of the radio-source statistics that are of particular cosmological significance: (1) the counts on the sky as a function of intensity, and (2) the distribution on the sky. These two statistics describe the extragalactic radio-source background in its simplest terms. However, interpretation in the form of constraints on Universal evolution is far from simple, requiring data at other frequencies, optical identifications and redshifts in particular.

\section{THE SOURCE COUNTS}

Searches for discrete radio sources have been of two types - big-dish (filled-aperture) surveys for the brighter sources, and aperture-synthesis surveys for the fainter sources. The brighter sources are all known; the whole sky has been searched. For the fainter sources, the rapid increase of source surface density as intensity decreases means that adequate statistical samples can be obtained by sampling tiny fractions of the sky, provided that the distribution is uniform. From these different survey procedures, the surface density as a function of intensity ("radiosource count," "N(S)" or "Log N - Log S" curve), has been measured at many frequencies (Kellerman and Wall 1987). Figure 2 shows an example.

The counts differ dramatically from those expected in uniformly filled universes either of "Euclidean" geometry or of conventional relativistic geometry. The law for the former is a horizontal line in Figure 2; for the latter, it shows a monotonic drop from the brightest to the

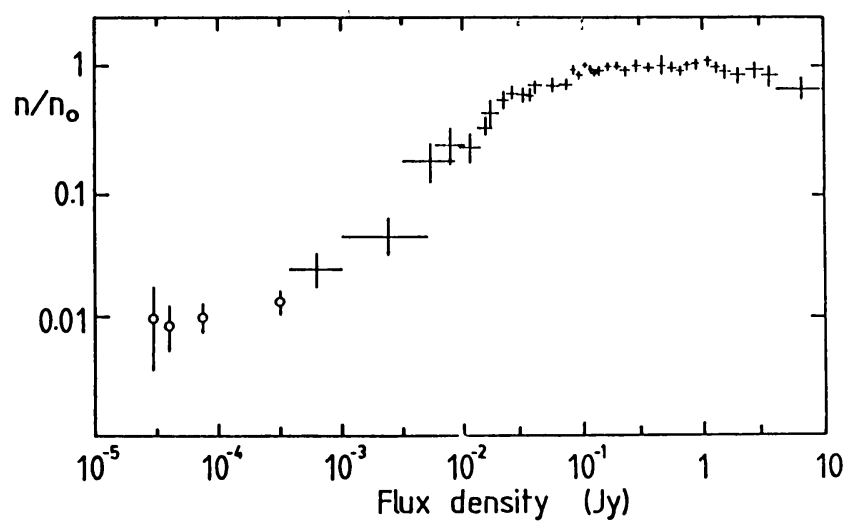

Figure 2. The source count at $5 \mathrm{GHz}$, normalized to that for a uniformly-filled static Euclidean universe with a differential number count of $n_{o}=90 \mathrm{~S}^{-5 / 2}$; as compiled by Kellermann and Wall (1987; see references therein) but with the addition of data at the lowest flux densities (open circles) from Fomalont et al. (1988). faintest intensities (see e.g., Figure 1 of Wall 1983). Radio sources must have been more powerful, or more numerous, or both, at the earlier epochs. In the face of identification and redshift data for many different samples of radio sources, it is not a tenable argument to claim that evolution can be dispensed with by some combination of statistical fluctuation ("local hole") and arbitrarily tiny redshifts for unidentified sources.

There have been many attempts to elucidate the details of this evolution; 
Wall (1983) discussed the incentives to do so and the need for a combined radio-optical approach. By then, the agreed general features of the evolution were: (a) that co-moving densities for the most powerful radio sources exceeded present-day values by factors of up to $10^{4}$ at epochs corresponding to $1<z<2$; (b) that a leveling-off or drop in this co-moving density occurred looking to even earlier epochs, and (c) that only the more luminous radio sources participated in this evolution. Much more has now been revealed. For example: (1) Condon (1984a,b, 1989) described a "shell" model in which both powerful and weak radio sources evolve similarly; (2) Peacock (1985) and Wall and Peacock (1985) demonstrated the similar nature of the evolution of "steep-spectrum" (extended-structure) radio galaxies and "flatspectrum" (compact-structure) QSOs; (3) Peacock (1985) provided the first evidence of a redshift cutoff, or at least a decrease in the co-moving density of compact radio sources at epochs corresponding to redshifts above 3, and (4) Danese et al. (1987) showed that simple luminosity-evolution models can provide a satisfactory description of the source-count data. It is a feature of the more recent analyses such as that of Danese et al. that these consider further populations of active objects, in particular the nature and space distribution of the possible "new blue" galaxy population (Kron, Koo and Windhorst 1985) which may be responsible for the flattening of the source counts at the lowest flux densities (Figure 2). The analyses of Danese et al. (1987) and Condon (1989) disagree over the nature of the blue galaxies (starburst/interacting versus radio-loud spirals) but agree that whatever the nature, the population must evolve strongly with epoch.

The extensive measurements of source counts make possible direct estimates of the contribution of discrete sources to the integrated radio background at different frequencies. A calculation by $\mathrm{G}$. De Zotti and L. Toffolatti (personal communication) yields the following results: $408 \mathrm{MHz}, 2.6 \mathrm{~K} ; 1.4 \mathrm{GHz}, 0.09 \mathrm{~K} ; 2.5 \mathrm{GHz}, 0.02 \mathrm{~K}$. Although this contribution (Figure 1) is generally swamped in magnitude by the nonthermal radiation from our Galaxy or by the $3^{\circ} \mathrm{MWBG}$, the fluctuations which the discrete-source background superposes on the MWBG must be considered in efforts to detect primordial MWBG fluctuations. Provided that sources are uniformly and randomly distributed on the sky, their fluctuation contribution can be estimated (Fomalont et al. 1988; Franceschini et al. 1989). Moreover, standard cosmological interpretations of source counts rest upon uniform sky distribution. Just how uniform is it?

\section{THE SURFACE DISTRIBUTION}

\subsection{The Background of Bright Radio Sources}

Efforts to identify optical counterparts and to obtain redshifts have resulted in virtually complete knowledge of the nature of all the bright radio sources. For the most part, they comprise QSOs and radio galaxies of very high optical and radio luminosities (see, e.g., Wall and Peacock 1985), although some nearby radio galaxies are also present in the mix.

With the completion of the first large-area centimeter-wavelength surveys, some nonuniformities appeared in the large-scale distribution of the brighter sources on the sky (Wall 1977, Pauliny-Toth 1977), anomalies which were distressingly close to statistical significance. Figure 3a shows one such result, a comparison of the source counts from the first 2 sr of the Parkes 2.7-GHz survey and from the next 5 sr. However, all formal analyses (e.g., binning, power-spectrum) have failed to find significant evidence against a uniform, random and independent surface distribution (Webster 1977). Eventually most of us, with differing degrees of reluctance, dismissed results such as Figure $3 \mathrm{a}$ as statistical accidents. 

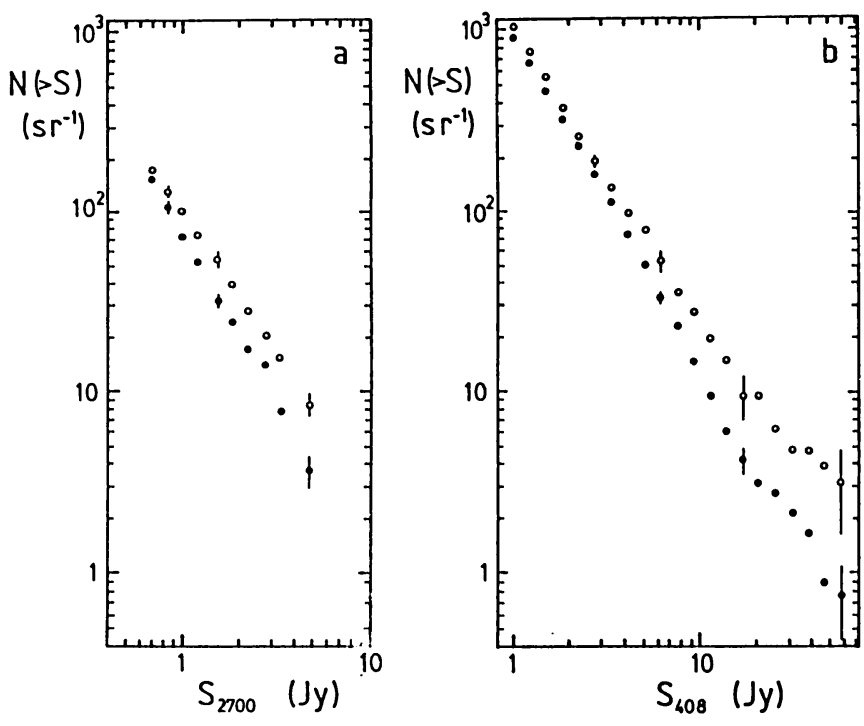

Figure 3. (a)Integral source counts for the first 2 sr of the Parkes 2.7-GHz survey (upper curve; maximum likelihood slope $-1.48 \pm 0.08)$ and the next 5 sr (slope $-1.91 \pm 0.06)$. (b) Integral source counts for the Molonglo 408-MHz survey; upper curve supergalactic latitude $<10^{\circ}$, lower curve supergalactic latitude $>10^{\circ}$.

Shaver and Pierre
(1989) were not prepared
to do so. Prompted by the
suggestion of major non-
uniformities in Universal
expansion due to the Great
Attractor, their analysis
showed convincingly that
the surface density of the
brighter radio sources is
markedly higher in the
supergalactic plane (Figure
3b). Many of the very
brightest sources are essen-
tially local ( $z$ < 0.05), too
close to partake in the
violent cosmological evolu-
tion; and when included in
a source count, they tend to
make it closer to Euclidcan.
This is particularly the case
if the integral form is used,
as in Figure 3b. Shaver
(personal communication)
has pointed out to me that
the first zones of the Parkes
2.7 GHz survey happened to be at low supergalactic latitudes; and it is this which produced the significant difference between the two counts of Figure 3a. For the Wall and Peacock (1985) all-sky sample, Shaver and Pierre found that the clustering to the supergalactic plane of the bright sources with $z<$ 0.02 is very marked. This in itself goes far towards explaining (a) differences between the counts of sources in different Galactic hemispheres (Pauliny-Toth 1977) and (b) differences between the forms of the source counts at high flux densities for flat and steep-spectrum radio sources noted by Wall and Peacock.

By explaining surface-distribution anomalies, the Shaver and Pierre result strengthens the basic interpretation of source counts in terms of active-galaxy evolution, but it demonstrates the importance of getting the right count to interpret; the very local objects need to be removed. In addition, it raises a basic question - why are the local radio galaxies confined to the local supercluster? This may be only a sampling effect; but it bears investigation.

An increase in the strength of the signal obtained by Shaver and Pierre may be achievable by removing the flat-spectrum sources, which are generally QSOs far beyond the local supercluster. There are other simple luminosity indicators in extragalactic radio sources (e.g., shape of the radio spectrum; radio structure) which could be used with large samples of sources from the major radio catalogues to examine surface distribution in different redshift shells. In this way, large structures other than the local supercluster may be traceable in redshift ranges which are barely accessible at other wavelengths. 


\subsection{The Background of Faint Radio Sources}

Only a small proportion of the sky has been searched for faint radio sources. Interpretation of the statistics for cosmological purposes depends therefore on small areas being typical - and the story for the bright sources provides a salutary tale. Nevertheless, effort devoted to finding differences between the aperture-synthesis surveys has generally been unsuccessful.

The faint radio sources - those selected in such aperture synthesis surveys - comprise QSOs and radio galaxies similar to those which host the bright sources, plus, at the faintest flux densities, a substantial number of blue galaxies whose nature remains the subject of

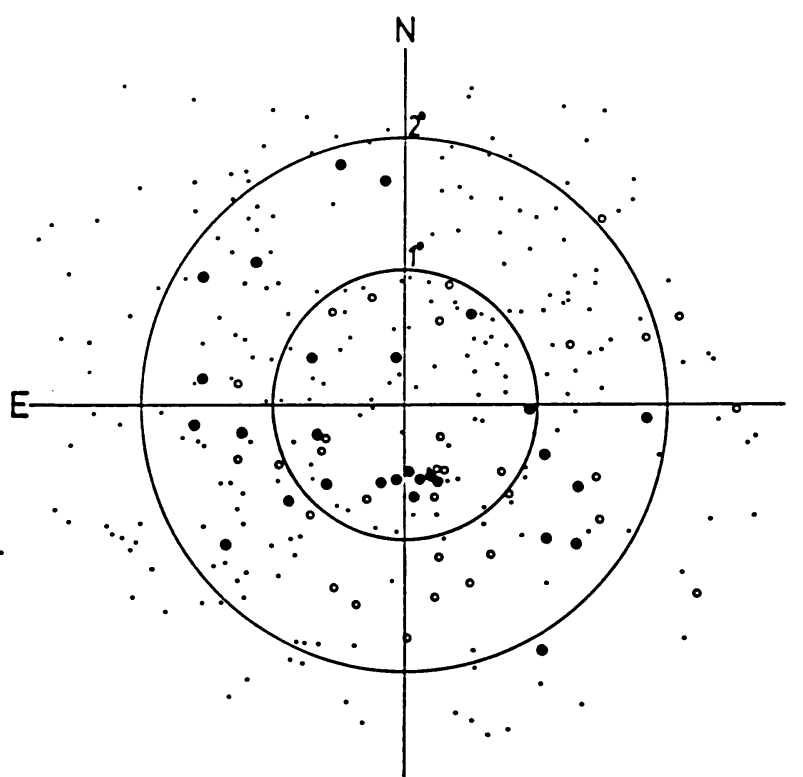

Figure 4. The distribution of radio sources on the sky from the 5C12 aperture-synthesis survey of Benn et al. (1982). Circles represent sources in a 10-50 $\mathrm{mJy}$ subsample for which identifications have been sought on deep (I $<22 \mathrm{mag}$ ) exposures taken on the $2.5 \mathrm{~m}$ Isaac Newton Telescope and with the $4.2 \mathrm{~m}$ William Herschel Telescope. Sources within the sample which have reliable identifications are indicated by filled circles. Note the possible supercluster 50 arcmin below the field center. debate. Benn et al. (1988) showed that disentangling the populations is feasible using accurate broad-band colors, and that the faint red galaxies identified in the $5 \mathrm{C} 12$ sample were very similar to the giant elliptical galaxies of the bright radio-source samples. Rixon, Benn and Wall (1989) demonstrated how colors and magnitudes for these objects may be used to provide redshift estimates accurate (for objects out to $z=0.5$ ) to 0.025 , opening the way for exploration of space distribution without spectroscopic redshifts. In fact, armed with right ascension, declination and apparent magnitude alone, it is possible to see structure emerging in the spatial distribution of the $5 \mathrm{C} 12$ radio sources (Figure 4). The Figure shows a clump of seven objects $30^{\prime}$ in extent some $40^{\prime}$ south of the field center. Six of the objects have magnitudes and (R-I) colors of elliptical galaxies at a redshift of about

0.8 , implying $\left(H_{o}=50 \mathrm{~km} \mathrm{sec} \mathrm{Mpc}^{-1}, \Omega=1\right.$ ) a projected largest extent of $15 \mathrm{Mpc}$. Have we detected a supercluster? Redshift measurements of these objects (at $R=20$ to 22 mag) would tell us. The seventh identification in this group has blue power-law colors and is almost certainly a QSO. Provided that it lies at a high enough redshift to place it beyond the putative supercluster and to move Lyman- $\alpha$ into the visible, a single deep spectrum to examine the Lyman- $\alpha$ forest could confirm the presence of a supercluster. 


\section{CONCLUSIONS ON THE BACKGROUND}

It can no longer be comfortably assumed that the discrete radio source background is uniformly and randomly distributed. Use of source-count statistics to determine cosmological distribution will in future require removal of the local supergalactic component; counts of sources on the basis of simple Poisson statistics may be misleading. Generalizations on the population mix based on one small area may be dangerous. Models of point-source contribution to the MWBG may need revision.

In return, however, the anisotropic radio-source background offers an exciting prospect: the mapping of large-scale structures in the Universe. The brightest sources show the presence of the local supercluster; the faintest may contain the imprint of distant superclusters. The middle ground remains to be explored. With some further analysis, optical identifications and photometry, we may be able to sketch out three-dimensional atlases of large chunks of the Universe.

Acknowledgments - I am grateful to Chris Benn, Gianfranco De Zotti, Jim Peebles, Guy Rixon and Peter Shaver and for stimulating discussions about it all, and to Chris Benn again for his helpful criticism of my manuscript.

\section{REFERENCES}

Benn, C. R., Grueff, G., Vigotti, M. and Wall, J. V. 1982, Mon Not. R. astr. Soc., 200, 747.

Benn, C. R., Grueff, G., Vigotti, M. and Wall, J. V. 1988, Mon. Not. R. astr. Soc., 230, 1.

Condon, J. J. 1984a, Astrophys. J., 284, 44.

Condon, J. J. 1984b, Astrophys. J., 287, 461.

Condon, J. J. 1989, Astrophys. J., 338, 13.

Danese, L., De Zotti, G., Franceschini, A., and Toffolatti, L. 1987, Astrophys. J., 318, L15.

Fomalont, E. B., Kellermann, K. I., Anderson, M. C., Weistrop, D., Wall, J. V., Windhorst, R. A., and Kristian, J. A. 1988, Astron. J., 96, 1187.

Franceschini, A., Toffolatti, L., Danese, L., and De Zotti, G. 1989. Astrophys. J., in press.

Kellermann, K. I. and Wall, J. V. 1987, in Observational Cosmology, Proc. IAU Symp. 124, eds. A. Hewitt and G. Burbidge, Dordrecht: Reidel, 545.

Kron, R. G., Koo, D. C., and Windhorst, R. A. 1985, Astron. Astrophys., 146, 38.

Pauliny-Toth, I. I. K. 1977, in Radio Astronomy and Cosmology, Proc. IAU Symp. 74, ed. D. L. Jauncey, Dordrecht: Reidel, 63.

Peacock, J. A. 1985, Mon. Not. R. astr. Soc., 217, 601.

Penzias, A. A. and Wilson, R. W. 1965, Astrophys. J., 142, 419.

Rixon, G. T., Benn, C. R., and Wall, J. V. 1989, "The intrinsic colour of radio-galaxies and photometric determination of redshift," Mon. Not. R. astr. Soc., submitted.

Shaver, P. A., and Pierre, M. 1989, Astron. Astrophys., in press.

Wall, J. V. 1977, in Radio Astronomy and Cosmology, Proc. IAU Symp. 74, ed. D. L. Jauncey, Dordrecht: Reidel, 55.

Wall, J. V. 1983, in The Origin and Evolution of Galaxies, NASI, eds, B. T. Jones and J. E. Jones, Dordrecht: Reidel, 295.

Wall, J. V., Chu, T. Y., and Yen, J. L. 1970, Austr. J. Phys., 23, 45.

Wall, J. V., and Peacock, J. A. 1985, Mon. Not. R. astr. Soc., 216, 173.

Webster, A. 1977, in Radio Astronomy and Cosmology, Proc. IAU Symp. 74, ed. D. L. Jauncey, Dordrecht: Reidel, 75. 